\title{
Stormwater wetlands for the enhancement of environmental ecosystem services: case studies for two retrofit wetlands in Brisbane, Australia
}

\author{
Margaret Greenway* \\ Environmental Engineering and Environmental Futures Research Institute, Griffith University, Nathan Campus, Brisbane, 4111 Queensland, Australia
}

\section{A R T I C L E I N F O}

\section{Article history:}

Received 4 March 2015

Received in revised form

16 December 2015

Accepted 22 December 2015

Available online $\mathrm{xxx}$

\section{Keywords:}

Ecosystem services

Irreducible background concentrations

Macroinvertebrates

Nutrients

Stormwater wetlands

Suspended solids

\begin{abstract}
A B S T R A C T
Constructed stormwater wetlands are examples of 'blue-green' ecological engineering in the urban environment providing a range of ecosystem services. A study was undertaken in Brisbane, Australia to examine the enhancement of environmental ecosystem services in 2 retrofit wetlands. Originally concrete drainage channels in existing residential catchments, a series of wetlands and ponds were created i.e. green infrastructure in a brownfield setting. Boardwalks, pathways and interpretive signage were incorporated into the design.

Golden Pond wetland was only $0.2 \%$ of the catchment whereas Bridgewater Creek - Bowie's Flat wetland was $0.4 \%$ of the catchment. The larger 0.8 ha Bridgewater Creek wetland had an overflow bypass channel. Water quality (suspended solids and nutrients) and the diversity of aquatic macroinvertebrates were monitored for 2-4 years after construction.

During storm events suspended solids increased at Golden Pond due to resuspension from the high velocities in this small linear flow system. Resuspension was minimised at Bridgewater Creek due to the by-pass channel. During dry weather suspended solids in both wetlands increased due to resuspension from the activity of waterbirds. Both wetlands were effective in reducing nutrient concentrations especially $\mathrm{NO}_{\mathrm{x}}-\mathrm{N}$ and $\mathrm{PO}_{4}-\mathrm{P}$, attributed to biological uptake; but during dry periods $\mathrm{NH}_{4}-\mathrm{N}$ increased within the system. The concept of irreducible background concentrations in wetland systems is discussed.

Open water and aquatic vegetation provided habitats for macroinvertebrates. Species richness increased by more than $50 \%$ in both stormwater wetlands, compared to the concrete drains.

Wetland sizing and design are essential parameters for water quality improvement and biodiversity.
\end{abstract}

(c) 2016 Elsevier Ltd. All rights reserved.

\section{Introduction}

Urbanisation decreases both green (forests and fields) and blue (wetlands and water bodies) space and increases impervious surface area so that rainfall infiltration capacity is reduced and the volume of stormwater runoff is increased. This leads to higher flood peaks in urban waterways with possible flooding and higher loads (concentrations and mass) of contaminants: sediment, nutrients, metals, hydrocarbons, organic micro -pollutants i.e. xenobiotics, pathogens. Many of these contaminants are primary stressors of aquatic ecosystem health (Greenway, 2004; Kadlec and Wallace, 2009; Hvitved-Jacobsen et al., 2010).

\footnotetext{
* Tel.: +61 737355296

E-mail address: m.greenway@griffith.edu.au.
}

Whilst the potential impacts of stormwater runoff, as a nonpoint source of pollution on water quality (and potential ecosystem health) have been recognised since the 1980's, legislation on stormwater control and the implementation of best management practices (BMP's) were not widely recognised and accepted until the 1990's (US EPA, 1996, 2000; Roy et al., 2008; Hvitved-Jacobsen et al., 2010). In USA best management practises are referred to as low impact development (LID), in Australia as water sensitive urban design (WSUD) and in UK as sustainable urban drainage systems (SUDS).

In Australia the first guidelines for Water Sensitive Urban Design (WSUD) were in Western Australia (Whelans et al., 1994). In Queensland, Brisbane City Council released similar guidelines in 1999 (BCC, 1999a,b). Brisbane City Council (2000) set stringent Water Quality Objectives: $15 \mathrm{mg} \mathrm{L}^{-1} \mathrm{TSS}, 5 \mathrm{mg} \mathrm{L}^{-1} \mathrm{TVS}, 0.65 \mathrm{mg} \mathrm{L}^{-1}$ TN, $0.035 \mathrm{mg} \mathrm{L}^{-1} \mathrm{NH}_{4}-\mathrm{N}, 0.13 \mathrm{mg} \mathrm{L}^{-1} \mathrm{NO}_{3}-\mathrm{N}, 0.07 \mathrm{mg} \mathrm{L}^{-1} \mathrm{TP}$, 
$0.035 \mathrm{mg} \mathrm{L}^{-1} \mathrm{PO}_{4}-\mathrm{P}, 8 \mu \mathrm{g} \mathrm{L}^{-1}$ chlorophyll-a; to meet legislative requirements to improve the quality of stormwater runoff which ultimately flows into Moreton Bay, designated in 1993 as a RAMSAR wetland/marine park with significant fisheries values and international ecological values (Ramsar Information Sheet: Moreton Bay).

Structural BMPs include: Gross Pollutant Traps (to catch coarse sediment and trash), Retention/Detention/Sediment Basins (to capture coarse and fine sediment, and removal of soluble contaminants), Vegetation Buffer Strips (removal by sheet flow across wide natural vegetation strips), Infiltration and Bioretention Systems (removal by physical filtration, chemical and biological processes), Vegetation Filter Strips/Grass Swales (removal along concentrated flow paths), Water Quality Control Ponds/Wet Ponds/ Dry Ponds/Wetlands (aquatic ecosystems - contaminant removal assisted by wetland plants, algae and microorganisms) (BCC, 1999a,b; US EPA, 2000; Hvitved-Jacobsen et al., 2010). Although the primary purpose of BMP's was seen as hydrological-controlling runoff flows, flood mitigation, water storage and contaminant removal to improve downstream water quality, it is now recognised that BMP's can be an infrastructure opportunity for the improvement of environmental (habitat and landscape enhancement, biodiversity, ground water protection) and social (recreational amenities, aesthetics, sustainable living) services in urbanised areas (Martin et al., 2007; Roy et al., 2008; Marsalek and Schreier, 2009; Hvitved-Jacobsen et al., 2010; Barbarosa et al., 2012).

Wetlands and ponds are suitable BMP's for both water storage and water quality improvement. Vegetation (emergent aquatic plants) is the dominant feature of wetlands, whereas open water is the dominant feature of ponds (Greenway et al., 2007). Ponds, which include sediment basins, retention/detention ponds are often deeper than $1.5 \mathrm{~m}$ and emergent plants are restricted to the shallow littoral margins. Submerged pondweeds may occur if there is sufficient light. The design of stormwater wetlands usually incorporates both zones of dense vegetation (shallow macrophytes zones) and deeper open water (Greenway, 2004; Kadlec and Wallace, 2009). The 'treatment' of stormwater as it flows through a wetland/pond is the result of complex interactions between physical, chemical and biological processes (Greenway, 2004; Kadlec and Wallace, 2009; Hvitved-Jacobsen et al., 2010). Vegetation plays an important role in these processes.

Ponds and wetlands also provide habitats for a range of biota, aquatic plants and animals as well as terrestrial wildlife (Wetzel, 2001; Mitsch and Gosselink, 2007; Cereghino et al., 2014). When constructed in residential areas to treat stormwater these water bodies can potentially balance the detrimental effects of urbanisation on predevelopment landscapes and ecosystems, achieving and sustaining biodiversity. Only recently have studies assessed these ecological values in stormwater wetlands (Woodcock et al., 2010; Tixier et al., 2011; Moore and Hunt, 2012, Herrmann, 2012).

This paper examines the environmental services of water quality improvement and aquatic macroinvertebrate biodiversity provided by 2 stormwater wetlands constructed within existing older residential areas ie new 'green and blue 'infrastructure within old 'brownfield' settings. Both wetlands were created from retrofitting concrete drainage channels. Social values are briefly discussed.

\section{Materials and methods}

\subsection{Wetland descriptions}

Two urban stormwater wetland systems were selected with similar catchment size (180 ha) to monitor water quality treatment and aquatic macroinvertebrate biodiversity. Both systems are located in Brisbane, SE Queensland; Australia. The climate is subtropical with an average annual rainfall of $1120 \mathrm{~mm}$. The wet season occurs over summer/autumn, with an average of 10days with greater than $50 \mathrm{~mm}$ daily rainfall; intense rainfall events of $1 \mathrm{~h}$ duration of $71 \mathrm{~mm} / \mathrm{h}$ are common. During the study period a 1 in 100 year event occurred when $208 \mathrm{~mm}$ fell in $3 \mathrm{~h}$.

The significance of the wetlands selected is that they are both 'retrofit' systems. They were designed and constructed within existing residential estates (brownfield sites) using sections of concrete channelised streambeds.

\subsubsection{Golden Pond stormwater treatment wetland system}

The Golden Pond system is located in Calamvale-a new (1990's) outer suburb SW of Brisbane. Prior to development three stream channels (tributaries of Calamvale Creek) fed into a series of lagoons with fringing riparian wetlands - known as the Kameruka Wetlands. The main north-south flowing stream tributary has a catchment of 160 ha, whereas the west-east flowing stream tributaries have a combined catchment of 50 ha.

As a consequence of residential development, considerable modification occurred. Initially the main (north-south flowing) stream was channelised into a concrete lined trapezoidal channel. However in 1999 Brisbane City Council decided to 'retrofit' the lower section $(120 \mathrm{~m})$ of the concrete channel into a 'linear flow wetland system' including a small sediment basin $(20 \mathrm{~m} \times 13 \mathrm{~m})$, a shallow wetland (Wetland $180 \mathrm{~m} \times 20 \mathrm{~m}$ )and a deeper pond (Wetland2 $50 \mathrm{~m} \times 20 \mathrm{~m}$ ) (Fig. 1). Pathways and a picnic shelter in parkland adjacent to Wetland 2 were incorporated into the design. Interpretive signage was erected to increase public awareness of stormwater quality improvement devices (SQIDs).

Stormwater from the concrete sediment basin flows into Wetland 1 (the Wetland) via a v-notched weir. Base flow and storm event discharge rates are given in Table 1. Discharge rates calculated for stormwater leaving the sediment basin and entering Wetland 1 ranged from 3 to $5.7 \mathrm{~m}^{3} \mathrm{~s}^{-1}$ for extreme (>20 y ARI) storm events, and from 0.15 to $0.8 \mathrm{~m}^{3} \mathrm{~s}^{-1}$ for high-intensity rain squalls. At discharge rates greater than $0.45 \mathrm{~m}^{3} \mathrm{~s}^{-1}$, short-circuiting occurs through the middle due to the positioning of a single V-notch weir, the lack of dense emergent macrophytes, and the linear nature of the flow path through the wetland. There is no bypass channel.

Between Wetland 1 (the Wetland) and Wetland 2 (the Pond), the water flows over a wide concrete sill. Piped underground drainage from the western section of residential development (20 ha) passes through a below ground Gross Pollutant Trap (GPT): CDS Proprietary Unit, before being discharged into Wetland2. Water then flows into Calamvale Creek.

The narrow outlet ( $1 \mathrm{~m}$ width) at the end of Wetland 2 ensures that the water backs up, thereby increasing the retention time. Water storage capacity and detention times are given in Table 1. It has been estimated that the average retention time for both wetlands during non-extreme storm events would be between 3 and $5 \mathrm{~h}$, and between 5 and $32 \mathrm{~h}$ for less intense rainfall events.

The total area of this 'constructed wetland system' is $3000 \mathrm{~m}^{2}$ i.e. $0.2 \%$ of the watershed catchment.

Piped drainage from the south western section of residential development (30 ha) also passes through a below ground GPT: ECOSOL Proprietary Unit, before entering the upper reaches of Calamvale Creek within a densely vegetated riparian wetland.

Calamvale Creek, several lagoons and adjacent riparian vegetation - the Kameruka Wetlands were retained in their natural state for a length of $680 \mathrm{~m}$ (Fig. 1). Calamvale Creek flows into Scrubby Creek, then the Logan River and into Moreton Bay Ramsar Wetland.

\subsubsection{Bridgewater Creek stormwater treatment wetland system:} Bowie's Flat Wetland

The Bridgewater Creek system is located at Cooparoo (SE Brisbane). Urbanisation of this suburb and adjacent Camp Hill occurred 


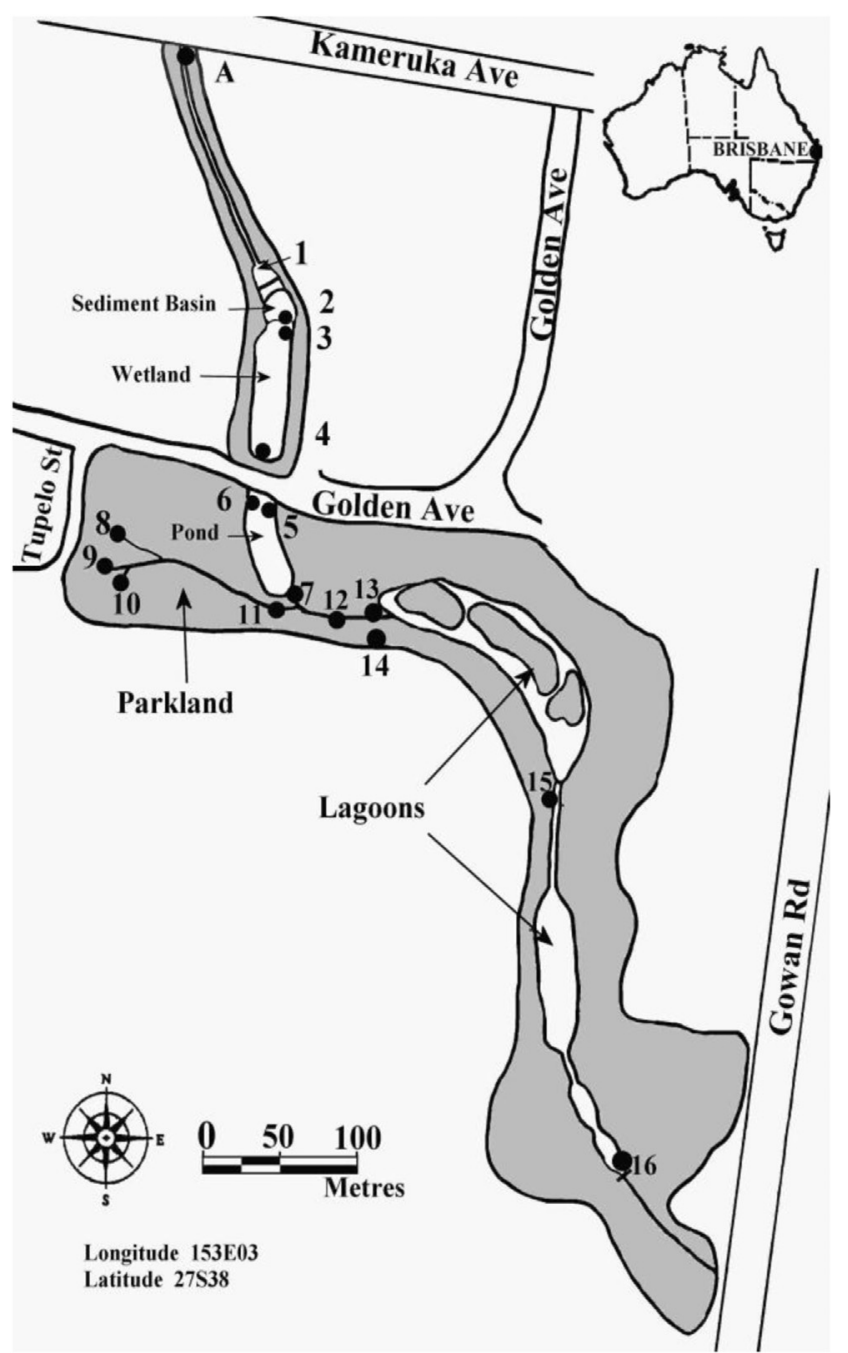

Fig. 1. Location Map of Sampling Sites (1-16) at Golden Pond Stormwater Treatment System (sediment basin; wetland $=$ Wetland1; pond $=$ Wetland2) and Kameruka Wetlands (Lagoons), Calamvale, Brisbane.

prior to 1950, when the original Bridgewater Creek was realigned and channelised to form a concrete drain. Bridgewater Creek flows into Norman Creek, then into the Brisbane River and into Moreton Bay Ramsar Wetland.

The Bridgewater Creek stormwater treatment wetland was constructed in Bowie's Flat parkland (an area of 1.5 ha). The concrete drain and adjoining grassy open space were excavated for the wetland basin ( $0.8 \mathrm{ha}$ ). While water quality improvement was the primary objective of this wetland, a picnic shelter, boardwalks and pathways for enhanced community visitations and recreation were incorporated into the design (Water by Design-Bridgewater Creek Wetland). Interpretive signage was erected to increase public awareness of the value of wetlands for water quality improvement and to enhance biodiversity.

Construction was completed in October 2001. This wetland is locally known as Bowie's Flat Wetland.

The "wetland" system consists of six inter-connected ponds and receives stormwater from two tributaries (Fig. 2-inlets). The main south-north flowing stream channel (Bridgewater Creek) has a catchment of 140 ha, whereas the piped drainage system from the west collects runoff from a 40 ha catchment. Before entering the "wetland", the stormwater runoff from both tributaries passes through trash racks, i.e. above-ground GPT, to collect trash and organic debris (leaf litter). The stormwater then passes into a sediment basin (Pond 1 ).

Pond 1, the sediment basin, is triangular in shape, with a surface area of $1000 \mathrm{~m}^{2}$, a depth of $2 \mathrm{~m}$ and a volume of $2000 \mathrm{~m}^{3}$ (standing water level) with a $2500 \mathrm{~m}^{3}$ capacity (during storm events). It has a fringing littoral zone of sedges.

Ponds 2 to 6 are oval-shaped, with dimensions of $40-50 \mathrm{~m}$ in length and $15-20 \mathrm{~m}$ in width; $0.2-2 \mathrm{~m}$ depth. They were originally designed as "macrophyte zones" to include open water, deep marsh, shallow marsh and ephemeral zones. Unfortunately, the establishment of wetland vegetation has been poor and is now largely restricted to narrow fringes along the shallow edges of the ponds (Greenway et al., 2007; Hunt et al., 2011).

Ponds 2-6 have a combined surface area of $7000 \mathrm{~m}^{2}$, and a volume of $4000 \mathrm{~m}^{3}$ (standing water level) and $10000 \mathrm{~m}^{3}$ (during storm events). The total area of this 'constructed wetland system' ('ponds' $1-6$ ) is $8000 \mathrm{~m}^{2}-0.4 \%$ of the watershed catchment.

Water flow from Pond 1 to Pond 2 occurs via an underground pipe, whereas surface water flow occurs progressively between Pond 2 to Pond 6. During periods of light rainfall and during dry weather, stormwater flows progressively through Ponds 1 to 6 . In times of heavy rainfall or storm events, the water levels rise rapidly so that Ponds 2 to 6 form a single lake-like water body. The outlet riser structure in Pond 6 determines stormwater residence time in the wetland and has been designed to ensure stormwater is retained in the wetland for at least $48 \mathrm{~h}$ optimum detention time for treatment determined by Brisbane City Council (BCC, 1999a,b). The retention times during wet weather range from $36 \mathrm{~h}$ for a major storm event to 6 days for less intense rainfall (Table 1 ). However at maximum ponding water level Bayley (2007) calculated a 9day extended detention time.

Table 1

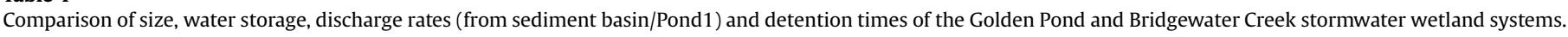

\begin{tabular}{|c|c|c|c|c|}
\hline & \multicolumn{2}{|c|}{ Golden pond ( $0.2 \%$ catchment) } & \multicolumn{2}{|l|}{ Bridgewater Creek (0.44\% catchment) } \\
\hline & Sediment basin & Wetland 1 and 2 'wetland' \& 'pond' & Pond 1 sediment basin & Ponds 2-6 'wetlands' \\
\hline Vegetation & Absent & $\begin{array}{l}\text { water lilies, aquatic creepers, } \\
\text { submerged pond weeds }\end{array}$ & $\begin{array}{l}\text { littoral/marsh emergent sedges submerged } \\
\text { pond weeds in pond } 6\end{array}$ & \\
\hline Area & $284 \mathrm{~m}^{2}$ & $2600 \mathrm{~m}^{2}$ & $1000 \mathrm{~m}^{2}$ & $7000 \mathrm{~m}^{2}$ \\
\hline Depth & $1.5 \mathrm{~m}$ & $0.2-1.2 \mathrm{~m}$ & $2 \mathrm{~m}$ & $0.2-1.5 \mathrm{~m}$ \\
\hline $\begin{array}{l}\text { Volume: Standing } \\
\text { Water Level }\end{array}$ & $100 \mathrm{~m}^{3}$ & $1300 \mathrm{~m}^{3}$ & $2000 \mathrm{~m}^{3}$ & $4000 \mathrm{~m}^{3}$ \\
\hline $\begin{array}{l}\text { Storm event } \\
\text { Discharge rates: }\end{array}$ & $150 \mathrm{~m}^{3}$ & $2000 \mathrm{~m}^{3}$ & $2500 \mathrm{~m}^{3}$ & $10,000 \mathrm{~m}^{3}$ \\
\hline Storm events & \multicolumn{2}{|c|}{$4.22-0.15 \mathrm{~m}^{3} \mathrm{~s}^{-1}$} & \multicolumn{2}{|l|}{$2.33-0.33 \mathrm{~m}^{3} \mathrm{~s}^{-1}$} \\
\hline Base flow & \multicolumn{2}{|c|}{$0.0015-0.0003 \mathrm{~m}^{3} \mathrm{~s}^{-1}$} & \multicolumn{2}{|l|}{$0.0015-0.0003 \mathrm{~m}^{3} \mathrm{~s}^{-1}$} \\
\hline Detention: & \multicolumn{2}{|c|}{ 3-16 h storm event; } & \multicolumn{2}{|l|}{$36 \mathrm{~h}-6$ days storm event; } \\
\hline Base flow & \multicolumn{2}{|c|}{$16-20$ days dry base flow } & \multicolumn{2}{|l|}{$>20$ days dry base flow } \\
\hline
\end{tabular}




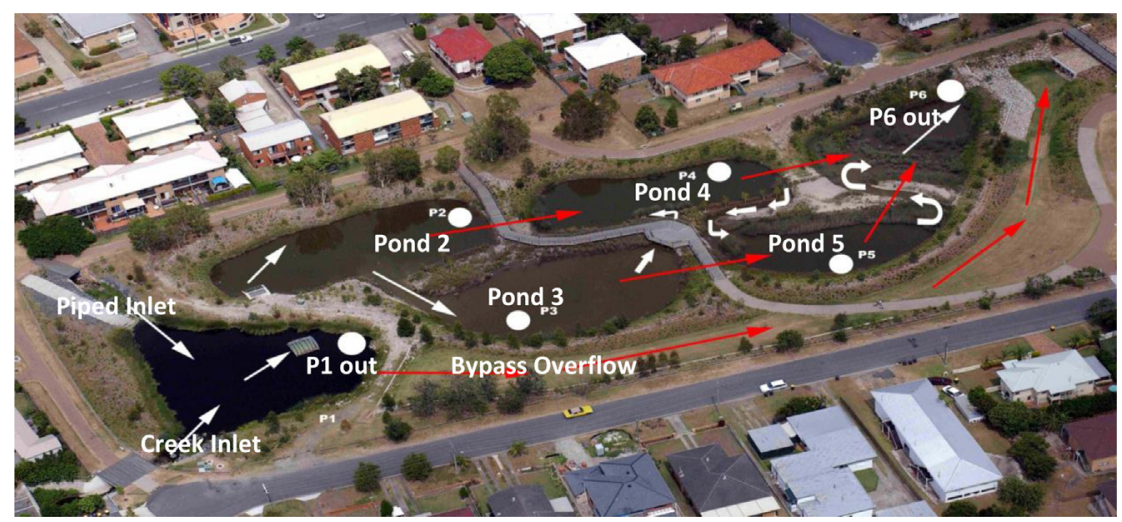

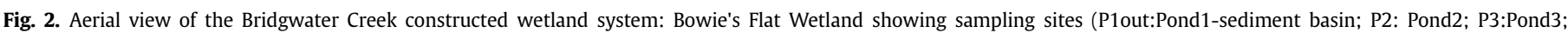

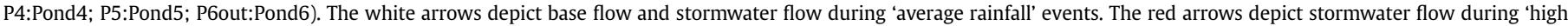
intensity rainfall' events. (For interpretation of the references to colour in this figure legend, the reader is referred to the web version of this article.)

During high-intensity storm events with high flow, the stormwater from Pond 1 can overflow via a spillway into a grassed "overflow bypass channel" which flows around the "wetland" and re-enters Bridgewater Creek flow path downstream of Pond 6 outlet. Bayley (2007) calculated that an intense $10 \mathrm{~mm}$ rainfall event in the catchment would generate runoff volumes greater than storage capacity and trigger the overflow from Pond1. The bypass is designed to protect the wetland vegetation from being damaged during high flow events and to reduce resuspension of the sediment. Since the overflow pathway is a grass swale it provides some sediment retention.

\subsection{Water quality monitoring}

Urbanised catchments with large impervious areas respond quickly to rainfall events. This often makes it difficult to interpret/ correlate water quality data with rainfall and runoff, unless synchronisation of sampling occurs. Due to the large number of sampling sites in these 2 systems automated samplers could not be installed, instead grab samples were taken. However, during storm events this presented difficulties since all sites could not be sampled simultaneously and many storms occurred at night. Thus 'storm event' samples were classified as "wet weather", with samples being collected within $12 \mathrm{~h}$ of the storm. Inter-event- base flow samples were classified as "dry weather". Data presented in this paper are from water quality monitoring over a 2 year period from 2002 to 2004.

At Golden Pond 6 Wet and 14 Dry weather water samples were collected. At Bridgewater Creek 20 Wet and 26 Dry weather samples were collected.

All water samples were analysed for Total Suspended Solids (TSS) and Total Volatile Solids (TVS) - the organic fraction; and nutrients: ammonium $\mathrm{NH}_{4}-\mathrm{N}$; nitrate-nitrite $\mathrm{NO}_{\mathrm{x}}-\mathrm{N}$; total nitrogen TN; phosphate $\mathrm{PO}_{4}-\mathrm{P}$ and total phosphorus TP. The analyses were carried out according to the standard methods (APHA-AWWA-WEF, 2005). TSS measurements followed procedure number $2540 \mathrm{D}$ (gravimetric, dried at $105{ }^{\circ} \mathrm{C}$ ) and TVS tests followed procedure number $2540 \mathrm{E}$ (gravimetric, ignited at $550{ }^{\circ} \mathrm{C}$ ). Nutrients were analysed using colorimetric methods with a Lachat Quikchem 8000 Flow Injection Analyser (FIA) (Hach Company, Loveland, Colorado). Subsamples for soluble nutrients were filtered with a $0.45-\mathrm{mm}$ filter paper before refrigeration. Total $\mathrm{N}$ and $\mathrm{P}$ were analysed following standard persulfate digestion on unfiltered samples.

Chlorophyll-a was measured (APHA-AWWA-WEF, 2005) as an indicator of phytoplankton activity.

\subsection{Macroinvertebrate monitoring}

Macroinvertebrates were sampled using a D-frame sweep net (0.5 mm mesh) which was alternately 'jabbed' into clumps of wetland vegetation and 'swept' through the water column over a distance of 1-2 m. Monitoring occurred over 2 years at Golden Pond and 4 years at Bridgewater Creek. Both summer (wet season) and winter (dry season) sampling was conducted. A $200 \mathrm{~mL}$ scoop was used to monitor the presence and abundance of any mosquito larvae (Greenway et al., 2003).

\section{Results}

\subsection{Water quality}

\subsubsection{Total suspended solids and Total Volatile Solids}

Tables 2 and 3 present both Wet and Dry weather data for the Golden Pond and Bridgewater Creek systems respectively. At Golden Pond the small sediment basin was not effective in removing TSS and TVS. At the bottom of Wetland1 TSS and TVS were higher than the stormwater both entering and leaving the sediment basin ie export; the increase in Wetland1 suggests resuspension. In storm events discharge rates from the V-notch weir in the sediment basin were as high as $4.22 \mathrm{~m}^{3} \mathrm{~s}^{-1}$ (Table 1). Wetland 2 removed some of the TSS but outflow concentrations were still higher than the stormwater inflows. Thus the Golden Pond constructed wetland system was not effective in removal of TSS and TVS during storm events.

The natural riparian wetland removed TSS from the Ecosol in Wet, but TSS increased in Dry due to an increase in organics-TVS. TSS in the Kameruka Wetlands showed large variation, but at $600 \mathrm{~m}$ downstream TSS decreased. In Dry TSS was lower than the inflow stormwater upstream. The proportion of TVS in Dry was consistently higher, and in the natural downstream channel made up to 75\%. The natural Kameruka Wetlands were more beneficial in TSS removal than the constructed wetlands in both storm events and base flow.

TSS and TVS in stormwater entering the Bridgewater Creek system showed large variations and was higher than stormwater runoff in the Golden Pond catchment. TSS leaving the sediment basin (Pond1 outlet) was lower than the stormwater inflows in both Wet and Dry i.e. there was removal. TVS was higher (70\%) in Dry possibly due to phytoplankton. In Wet TSS between Pond 1 and Pond 6 decreased with an overall removal of 27\%. However, in Dry TSS and TVS increased in Ponds 2 and 3 due to particulate organics. 
Table 2

TSS and TVS (mg/L mean \pm SD) Golden Pond Stormwater Treatment Train: ns (insignificant).

\begin{tabular}{|c|c|c|c|c|c|c|c|}
\hline Site & Description & Wet TSS & Wet TVS & \% Organics & Dry TSS & Dry TVS & $\%$ Organics \\
\hline 1 & In Sediment Basin & $17 \pm 12$ & $4 \pm 3$ & $41 \%$ & $6 \pm 4$ & $3 \pm 2$ & $50 \%$ \\
\hline \multirow[t]{2}{*}{2} & Out Sediment Basin & $20 \pm 8$ & $5 \pm 2$ & $25 \%$ & $6 \pm 3$ & $3 \pm 3$ & $50 \%$ \\
\hline & 1-2: Removal & ns & ns & & ns & ns & \\
\hline 3 & Top Wetland 1 & $22 \pm 9$ & $5 \pm 3$ & $23 \%$ & $7 \pm 4$ & $4 \pm 2$ & $57 \%$ \\
\hline \multirow[t]{2}{*}{4} & Bottom Wetland 1 & $26 \pm 10$ & $6 \pm 3$ & $23 \%$ & $14 \pm 6$ & $6 \pm 3$ & $43 \%$ \\
\hline & 3-4: Removal & EXPORT & ns & & EXPORT & EXPORT & \\
\hline 5 & Top Wetland 2 & 53 & 11 & $22 \%$ & $16 \pm 9$ & $8 \pm 7$ & $50 \%$ \\
\hline 6 & CDS S/W Outlet & $12 \pm 2$ & $4 \pm 2$ & $33 \%$ & $6 \pm 3$ & $4 \pm 2$ & $67 \%$ \\
\hline \multirow[t]{3}{*}{7} & Bottom Wetland 2 & $24 \pm 12$ & $5 \pm 3$ & $21 \%$ & $13 \pm 9$ & $7 \pm 5$ & $54 \%$ \\
\hline & 4-7: Removal \% & $8 \%$ & $17 \%$ & & $7 \%$ & ns & \\
\hline & 1-7: & EXPORT & EXPORT & & EXPORT & EXPORT & \\
\hline 9 & Ecosol Bypass & $23 \pm 9$ & $5 \pm 1$ & $22 \%$ & $7 \pm 5$ & $4 \pm 3$ & $57 \%$ \\
\hline 10 & Ecosol S/W Outlet & $18 \pm 8$ & $5 \pm 2$ & $28 \%$ & $12 \pm 9$ & $5 \pm 4$ & $42 \%$ \\
\hline \multirow[t]{2}{*}{11} & Riparian Wetland & $15 \pm 8$ & $4 \pm 2$ & $27 \%$ & $14 \pm 11$ & $7 \pm 5$ & $50 \%$ \\
\hline & 9-11: Removal \% & $35 \%$ & ns & & EXPORT & EXPORT & \\
\hline 12 & Confluence $7+11$ & $24 \pm 16$ & $5 \pm 4$ & $21 \%$ & $9 \pm 5$ & $6 \pm 4$ & $67 \%$ \\
\hline 13 & $100 \mathrm{~m}$ downstream & $29 \pm 9$ & $6 \pm 2$ & $21 \%$ & $6 \pm 4$ & $4 \pm 2$ & $67 \%$ \\
\hline 15 & $230 \mathrm{~m}$ downstream & $37 \pm 7$ & $10 \pm 4$ & $33 \%$ & $8 \pm 5$ & $6 \pm 4$ & $75 \%$ \\
\hline \multirow[t]{2}{*}{16} & $600 \mathrm{~m}$ downstream & $21 \pm 16$ & $5 \pm 4$ & $24 \%$ & $5 \pm 2$ & $3 \pm 3$ & $60 \%$ \\
\hline & 12-16:Removal \% & $13 \%$ & nil & & $44 \%$ & $50 \%$ & \\
\hline
\end{tabular}

Table 3

TSS and TVS (mg/L mean \pm SD) Bridgewater Creek Stormwater Treatment System.

\begin{tabular}{|c|c|c|c|c|c|c|}
\hline \multirow[t]{2}{*}{ Location } & \multicolumn{3}{|c|}{ Wet weather } & \multicolumn{3}{|c|}{ Dry weather } \\
\hline & TSS & TVS & $\%$ TVS & TSS & TVS & $\%$ TVS \\
\hline Creek Inlet & $66 \pm 40$ & $21 \pm 14$ & $32 \%$ & $18 \pm 17$ & $5 \pm 3$ & $28 \%$ \\
\hline Piped Inlet & $33 \pm 16$ & $10 \pm 4$ & $30 \%$ & $17 \pm 16$ & $5 \pm 3$ & $30 \%$ \\
\hline $\begin{array}{l}\text { Pond } 1 \text { out } \\
\text { IN-OUT P1 }\end{array}$ & $\begin{array}{l}41 \pm 34 \\
\text { Decrease }\end{array}$ & $9 \pm 5$ & $22 \%$ & $\begin{array}{l}10 \pm 6 \\
\text { Decrease }\end{array}$ & $7 \pm 5$ & $70 \%$ \\
\hline Pond 2 & $40 \pm 27$ & $11 \pm 5$ & $28 \%$ & $30 \pm 20$ & $18 \pm 12$ & $60 \%$ \\
\hline Pond 3 & $40 \pm 27$ & $14 \pm 8$ & $35 \%$ & $37 \pm 20$ & $27 \pm 17$ & $73 \%$ \\
\hline Pond 4 & $35 \pm 27$ & $15 \pm 6$ & $43 \%$ & $23 \pm 10$ & $16 \pm 7$ & $70 \%$ \\
\hline Pond 5 & $34 \pm 28$ & $13 \pm 8$ & $38 \%$ & $20 \pm 10$ & $12 \pm 7$ & $60 \%$ \\
\hline Pond 6 out & $30 \pm 26$ & $10 \pm 5$ & $33 \%$ & $16 \pm 8$ & $7 \pm 4$ & $44 \%$ \\
\hline P1-P6\%removal & $27 \%$ & & & Export & & \\
\hline IN-OUT P1 & Decrease & & & Decrease & & \\
\hline
\end{tabular}

Although some reduction occurred in Ponds 4 and 5, outflow from Pond 6 was still higher than from Pond1. However overall there was a slight decrease in TSS compared to the stormwater inflows. The proportion of TVS in Dry was consistently higher than in Wet suggesting either phytoplankton or resuspension of organic matter.

\subsubsection{Nutrients}

Tables 4-7 present Wet and Dry weather nutrient data for the Golden Pond and Bridgewater Creek systems respectively. Both systems show large variations in stormwater inflow nutrient concentrations in Wet and Dry, and variation within the different 'wetland components' however, mean outflow concentrations were consistently lower than stormwater entering the systems.

At Golden Pond in Wet there was negligible removal of nutrients into and out of the sediment basin; however significant removal of $\mathrm{NH}_{4}-\mathrm{N}, \mathrm{NO}_{\mathrm{x}}-\mathrm{N}$ and $\mathrm{PO}_{4}-\mathrm{P}$ occurred in Wetland1. Wetland 2 was highly effective in removing nutrients flowing into the wetland via the piped drainage system (CDS Stormwater Outlet) with up to $80 \%$ reduction in $\mathrm{NO}_{\mathrm{x}}-\mathrm{N}$ and $\mathrm{PO}_{4}-\mathrm{P}$ concentrations and 65\% reduction in TN and TP concentrations. The riparian wetland was also effective in reducing nutrient concentrations via the Ecosol Stormwater Outlet. The $600 \mathrm{~m}$ length of natural wetlands was effective in further nutrient reduction.

In Dry only a reduction in $\mathrm{PO}_{4}-\mathrm{P}$ concentrations occurred in the sediment basin; reductions of $\mathrm{NH}_{4}-\mathrm{N}, \mathrm{NO}_{\mathrm{x}}-\mathrm{N}$ and $\mathrm{PO}_{4}-\mathrm{P}$ occurred in Wetland1. Wetland2 was again highly effective in removing nutrients from the $\mathrm{S} / \mathrm{W}$ Outlet, though $\mathrm{NH}_{4}-\mathrm{N}$ was higher at

Table 4

Nutrients $(\mathrm{mg} / \mathrm{L}$ mean $\pm \mathrm{SD})$ : Golden Pond Stormwater Treatment Train: Wet Weather.

\begin{tabular}{|c|c|c|c|c|c|c|}
\hline Site & Description & $\mathrm{NH}_{4}-\mathrm{N}$ & $\mathrm{NO}_{\mathrm{x}}-\mathrm{N}$ & $\mathrm{TN}$ & $\mathrm{PO}_{4}-\mathrm{P}$ & $\mathrm{TP}$ \\
\hline 1 & Into Sed Basin & $0.10 \pm 0.10$ & $0.83 \pm 0.48$ & $1.27 \pm 0.63$ & $0.09 \pm 0.09$ & $0.11 \pm 0.05$ \\
\hline \multirow[t]{2}{*}{2} & Out Sed Basin & $0.09 \pm 0.09$ & $0.68 \pm 0.38$ & $1.22 \pm 0.58$ & $0.08 \pm 0.04$ & $0.12 \pm 0.05$ \\
\hline & 1-2: Removal\% & $10 \%$ & $18 \%$ & $4 \%$ & $11 \%$ & insignificant \\
\hline 3 & Top Wetland 1 & $0.12 \pm 0.10$ & $0.47 \pm 0.28$ & $1.01 \pm 0.28$ & $0.08 \pm 0.07$ & $0.11 \pm 0.02$ \\
\hline \multirow[t]{3}{*}{4} & Bottom Wetland 1 & $0.08 \pm 0.07$ & $0.43 \pm 0.46$ & $1.12 \pm 0.58$ & $0.06 \pm 0.06$ & $0.13 \pm 0.07$ \\
\hline & 3-4: Removal\% & $33 \%$ & $9 \%$ & export & $25 \%$ & insignificant \\
\hline & 1-4: Removal\% & $20 \%$ & $40 \%$ & $15 \%$ & $34 \%$ & \\
\hline 5 & Top Wetland 2 & $0.09 \pm 0.06$ & $0.25 \pm 0.40$ & 0.92 & $0.03 \pm 0.04$ & $0.09 \pm 0$ \\
\hline 6 & CDS S/W Outlet & $0.12 \pm 0.05$ & $1.92 \pm 1.47$ & $2.50 \pm 1.22$ & $0.24 \pm 0.15$ & $0.34 \pm 0.22$ \\
\hline \multirow[t]{2}{*}{7} & Bottom Wetland 2 & $0.07 \pm 0.04$ & $0.37 \pm 0.28$ & $0.94 \pm 0.51$ & $0.05 \pm 0.05$ & $0.11 \pm 0.06$ \\
\hline & 6-7: Removal\% & $42 \%$ & $80 \%$ & $62 \%$ & $79 \%$ & $65 \%$ \\
\hline 9 & Ecosol Bypass & $0.09 \pm 0.05$ & $0.53 \pm 0.60$ & NA & $0.07 \pm 0.06$ & 0.07 \\
\hline 10 & Ecosol S/W Outlet & $0.11 \pm 0.08$ & $1.07 \pm 0.68$ & $1.75 \pm 0.55$ & $0.10 \pm 0.09$ & $0.15 \pm 0.06$ \\
\hline \multirow[t]{2}{*}{11} & Riparian Wetland & $0.05 \pm 0.04$ & $0.61 \pm 0.45$ & 1.14 & $0.07 \pm 0.08$ & $0.13 \pm 0.04$ \\
\hline & 10-11: Removal\% & $55 \%$ & $43 \%$ & $35 \%$ & $30 \%$ & $13 \%$ \\
\hline 12 & Confluence $7+11$ & $0.07 \pm 0.03$ & $0.68 \pm 0.35$ & $1.16 \pm 0.46$ & $0.06 \pm 0.08$ & $0.13 \pm 0.04$ \\
\hline 13 & $100 \mathrm{~m}$ downstream & $0.13 \pm 0.01$ & $0.63 \pm 0.15$ & $1.22 \pm 0.29$ & $0.05 \pm 0.05$ & $0.10 \pm 0.02$ \\
\hline 15 & $230 \mathrm{~m}$ downstream & $0.12 \pm 0.03$ & $0.47 \pm 0.16$ & 0.71 & $0.03 \pm 0.04$ & $0.12 \pm 0.02$ \\
\hline \multirow[t]{2}{*}{16} & $600 \mathrm{~m}$ downstream & $0.06 \pm 0.03$ & $0.42 \pm 0.17$ & $0.92 \pm 0.27$ & $0.04 \pm 0.02$ & $0.09 \pm 0.03$ \\
\hline & 12-16: Removal\% & $14 \%$ & $38 \%$ & $21 \%$ & $33 \%$ & $31 \%$ \\
\hline
\end{tabular}


Table 5

Nutrients $(\mathrm{mg} / \mathrm{L}$ mean $\pm \mathrm{SD})$ : Golden Pond Stormwater Treatment Train: Dry Weather.

\begin{tabular}{|c|c|c|c|c|c|c|}
\hline Site & Description & $\mathrm{NH}_{4}-\mathrm{N}$ & $\mathrm{NO}_{\mathrm{x}}-\mathrm{N}$ & $\mathrm{TN}$ & $\mathrm{PO}_{4}-\mathrm{P}$ & TP \\
\hline 1 & Into Sed Basin & $0.03 \pm 0.03$ & $0.53 \pm 0.67$ & $0.57 \pm 0.31$ & $0.04 \pm 0.04$ & $0.08 \pm 0.02$ \\
\hline \multirow[t]{2}{*}{2} & Out Sed Basin & $0.05 \pm 0.05$ & $0.56 \pm 0.71$ & $0.70 \pm 0.33$ & $0.03 \pm 0.03$ & $0.08 \pm 0.01$ \\
\hline & 1-2: Removal\% & EXPORT & ns & EXPORT & $25 \%$ & nil \\
\hline 3 & Top Wetland 1 & $0.04 \pm 0.03$ & $0.43 \pm 0.63$ & $0.74 \pm 0.33$ & $0.03 \pm 0.03$ & $0.07 \pm 0.01$ \\
\hline \multirow[t]{3}{*}{4} & Bottom Wetland 1 & $0.03 \pm 0.03$ & $0.25 \pm 0.49$ & $0.63 \pm 0.33$ & $0.02 \pm 0.02$ & $0.07 \pm 0.02$ \\
\hline & 3-4: Removal\% & $25 \%$ & $42 \%$ & $15 \%$ & $33 \%$ & Nil \\
\hline & 1-4: Removal\% & nil & $53 \%$ & EXPORT & $50 \%$ & $13 \%$ \\
\hline 5 & Top wetland 2 & $0.06 \pm 0.09$ & $0.31 \pm 0.41$ & $0.70 \pm 0.28$ & $0.02 \pm 0.03$ & $0.09 \pm 0.02$ \\
\hline 6 & CDS S/W Outlet & $0.07 \pm 0.06$ & $1.76 \pm 0.78$ & $2.35 \pm 0.37$ & $0.11 \pm 0.06$ & $0.19 \pm 0.05$ \\
\hline \multirow[t]{2}{*}{7} & Bottom Wetland 2 & $0.08 \pm 0.09$ & $0.25 \pm 0.23$ & $0.97 \pm 0.50$ & $0.05 \pm 0.08$ & $0.14 \pm 0.07$ \\
\hline & 6-7: Removal\% & EXPORT & $85 \%$ & $\mathbf{5 9} \%$ & $55 \%$ & $26 \%$ \\
\hline 9 & Ecosol Bypass & $0.02 \pm 0.02$ & $0.30 \pm 0.51$ & $1.05 \pm 0.45$ & $0.01 \pm 0.01$ & $0.09 \pm 0.04$ \\
\hline 10 & Ecosol S/W Outlet & $0.17 \pm 0.36$ & $1.10 \pm 0.81$ & $1.53 \pm 0.79$ & $0.04 \pm 0.04$ & $0.08 \pm 0.02$ \\
\hline \multirow[t]{2}{*}{11} & Riparian Wetland & $0.26 \pm 0.43$ & $0.28 \pm 0.25$ & $0.84 \pm 0.25$ & $0.02 \pm 0.02$ & $0.09 \pm 0.05$ \\
\hline & 10-11: Removal\% & EXPORT & $82 \%$ & $45 \%$ & $50 \%$ & Insignificant \\
\hline 12 & Confluence $7+11$ & $0.09 \pm 0.06$ & $0.21 \pm 0.18$ & $0.75 \pm 0.32$ & $0.03 \pm 0.03$ & $0.08 \pm 0.04$ \\
\hline 13 & $100 \mathrm{~m}$ downstream & $0.06 \pm 0.06$ & $0.22 \pm 0.28$ & $0.59 \pm 0.30$ & $0.02 \pm 0.02$ & $0.06 \pm 0.02$ \\
\hline 15 & $230 \mathrm{~m}$ downstream & $0.07 \pm 0.06$ & $0.08 \pm 0.11$ & $0.61 \pm 0.21$ & $0.02 \pm 0.02$ & $0.05 \pm 0.04$ \\
\hline \multirow[t]{2}{*}{16} & $600 \mathrm{~m}$ downstream & $0.03 \pm 0.03$ & $0.09 \pm 0.11$ & $0.60 \pm 0.28$ & $0.02 \pm 0.02$ & $0.05 \pm 0.03$ \\
\hline & 12-16: Removal\% & $67 \%$ & $59 \%$ & $20 \%$ & $33 \%$ & $\mathbf{3 8} \%$ \\
\hline
\end{tabular}

Table 6

Nutrients: Bridgewater Creek treatment system: Wet weather (mean $\pm \mathrm{SD}$ ).

\begin{tabular}{|c|c|c|c|c|c|}
\hline Site & $\mathrm{NH}_{4}-\mathrm{N}(\mathrm{mg} / \mathrm{L})$ & $\mathrm{NO}_{\mathrm{x}}-\mathrm{N}(\mathrm{mg} / \mathrm{L})$ & $\mathrm{TN}(\mathrm{mg} / \mathrm{L})$ & $\mathrm{PO}_{4}-\mathrm{P}(\mathrm{mg} / \mathrm{L})$ & $\mathrm{TP}(\mathrm{mg} / \mathrm{L})$ \\
\hline Creek Inlet & $0.14 \pm 0.12$ & $0.43 \pm 0.17$ & $4.68 \pm 2.70$ & $0.14 \pm 0.16$ & $0.70 \pm 0.38$ \\
\hline Piped Inlet & $0.12 \pm 0.10$ & $0.42 \pm 0.32$ & $3.62 \pm 3.36$ & $0.10 \pm 0.09$ & $0.31 \pm 0.22$ \\
\hline Pond 1 Out & $0.13 \pm 0.11$ & $0.46 \pm 0.37$ & $2.69 \pm 3.12$ & $0.15 \pm 0.21$ & $0.21 \pm 0.19$ \\
\hline IN - OUT P1 & insignificant & insignificant & decrease & insignificant & decrease \\
\hline Pond 2 & $0.31 \pm 0.03$ & $0.13 \pm 0.07$ & $1.14 \pm 0.19$ & $0.04 \pm 0.02$ & $0.20 \pm 0.03$ \\
\hline Pond 3 & $0.10 \pm 0.10$ & $0.13 \pm 0.14$ & $1.84 \pm 0.87$ & $0.08 \pm 0.05$ & $0.30 \pm 0.14$ \\
\hline Pond 4 & $0.20 \pm 0.27$ & $0.16 \pm 0.10$ & $1.73 \pm 0.37$ & $0.04 \pm 0.03$ & $0.26 \pm 0.18$ \\
\hline Pond 5 & $0.28 \pm 0.06$ & $0.16 \pm 0.10$ & $1.42 \pm 0.31$ & $0.02 \pm 0.02$ & $0.19 \pm 0.06$ \\
\hline Pond 6 Out & $0.13 \pm 0.22$ & $0.10 \pm 0.08$ & $0.84 \pm 0.21$ & $0.02 \pm 0.02$ & $0.12 \pm 0.08$ \\
\hline P1-P6: Removal\% & insignificant & $78 \%$ & $69 \%$ & $87 \%$ & $43 \%$ \\
\hline
\end{tabular}

Table 7

Nutrients: Bridgewater Creek treatment system: Dry weather (mean \pm SD).

\begin{tabular}{|c|c|c|c|c|c|}
\hline $\mathrm{N}$ & $\mathrm{NH}_{4}-\mathrm{N}(\mathrm{mg} / \mathrm{L})$ & $\mathrm{NO}_{\mathrm{x}}-\mathrm{N}(\mathrm{mg} / \mathrm{L})$ & $\mathrm{TN}(\mathrm{mg} / \mathrm{L})$ & $\mathrm{PO}_{4}-\mathrm{P}(\mathrm{mg} / \mathrm{L})$ & $\mathrm{TP}(\mathrm{mg} / \mathrm{L})$ \\
\hline Creek Inlet & $0.08 \pm 0.09$ & $0.57 \pm 0.60$ & $1.84 \pm 1.01$ & $0.19 \pm 0.18$ & $0.26 \pm 0.11$ \\
\hline Piped Inlet & $0.06 \pm 0.05$ & $1.10 \pm 0.40$ & $1.97 \pm 0.28$ & $0.16 \pm 0.10$ & $0.24 \pm 0.09$ \\
\hline Pond 1 Out & $0.10 \pm 0.09$ & $0.12 \pm 0.24$ & $1.28 \pm 0.45$ & $0.08 \pm 0.06$ & $0.22 \pm 0.10$ \\
\hline IN-OUT P1 & increase & decrease & decrease & decrease & decrease \\
\hline Pond 2 & $0.22 \pm 0.30$ & $0.06 \pm 0.10$ & $1.46 \pm 0.35$ & $0.05 \pm 0.04$ & $0.27 \pm 0.06$ \\
\hline Pond 3 & $0.22 \pm 0.29$ & $0.07 \pm 0.17$ & $1.38 \pm 0.50$ & $0.04 \pm 0.03$ & $0.26 \pm 0.08$ \\
\hline Pond 4 & $0.15 \pm 0.23$ & $0.03 \pm 0.04$ & $1.14 \pm 0.35$ & $0.03 \pm 0.02$ & $0.23 \pm 0.07$ \\
\hline Pond 5 & $0.19 \pm 0.23$ & $0.08 \pm 0.15$ & $1.23 \pm 0.38$ & $0.02 \pm 0.02$ & $0.21 \pm 0.08$ \\
\hline Pond 6 Out & $0.11 \pm 0.11$ & $0.10 \pm 0.14$ & $1.04 \pm 0.36$ & $0.02 \pm 0.01$ & $0.17 \pm 0.07$ \\
\hline P1-P6 out & Export & $17 \%$ & $19 \%$ & $75 \%$ & $23 \%$ \\
\hline IN-OUT P6 & EXPORT & $82-91 \%$ & $44-47 \%$ & $88-90 \%$ & $35-29 \%$ \\
\hline
\end{tabular}

Wetland2 outlet. Similarly the riparian wetland reduced nutrients from the $\mathrm{S} / \mathrm{W}$ outlet but $\mathrm{NH}_{4}-\mathrm{N}$ increased. The downstream wetland provided further nutrient reduction including $\mathrm{NH}_{4}-\mathrm{N}$ (67\%).

Thus both the constructed wetland system and the natural wetland system were effective in nutrient removal from stormwater inflows.

At Bridgewater Creek in Wet, only TN and TP were consistently lower in Pond1 (sediment basin) compared to stormwater inflow concentrations. Whilst there was considerable variation in nutrient concentrations between Ponds2-5, including increases in $\mathrm{NH}_{4}-\mathrm{N}$, by the time the stormwater reached Pond6 outlet, there had been an overall reduction compared to Pond 1 outflow especially in $\mathrm{NO}_{\mathrm{X}}-\mathrm{N}(78 \%)$ and $\mathrm{PO}_{4}-\mathrm{P}(67 \%)$.
In Dry there was removal in Pond1, with the exception of $\mathrm{NH}_{4}-\mathrm{N}$. $\mathrm{NH}_{4}-\mathrm{N}$ (also reflected in TN) increased in Pond2 and although reductions did occur; outflow concentrations at P6 were greater than stormwater inflows, so the system exported $\mathrm{NH}_{4}-\mathrm{N}$ Concentrations of other nutrients were reduced, with removal of up to $90 \% \mathrm{NO}_{\mathrm{x}}-\mathrm{N}$ and $\mathrm{PO}_{4}-\mathrm{P}$, compared to stormwater inflows. Biological uptake by plants, algae and phytoplankton would have played a major role, especially given detention times of 20 days plus during dry periods.

\subsubsection{Phytoplankton}

Chlorophyll-a. At Golden Pond, chlorophyll-a was only measured in Dry base-flow periods and values were low $\left(3.5 \pm 0.6 \mu \mathrm{g} \mathrm{L}^{-1}\right.$ in the sediment basin, $5.5 \pm 3.2 \mu \mathrm{g} \mathrm{L}{ }^{-1}$ in Wetland 
Table 8

Chlorophyll-a ( $\left.\mu \mathrm{g} \mathrm{L}^{-1}\right)$ an indicator of phytoplankton biomass in the Bridgewater Creek System. Dry (base flow) and Wet (storm events). Mean \pm SD.

\begin{tabular}{|c|c|c|c|c|c|c|}
\hline & Pond 1 (sediment basin) & Pond 2 & Pond 3 & Pond 4 & Pond 5 & Pond 6 \\
\hline Dry & $64 \pm 80$ & $54 \pm 60$ & $15 \pm 20$ & $12 \pm 12$ & $10 \pm 5$ & $12 \pm 10$ \\
\hline Wet & $12 \pm 15$ & $22 \pm 30$ & $25 \pm 30$ & $22 \pm 28$ & $28 \pm 22$ & $33 \pm 20$ \\
\hline
\end{tabular}

1 , and $3.2 \pm 0.8 \mu \mathrm{g} \mathrm{L}^{-1}$ in Wetland 2). All values are below WQO $\left(8 \mu \mathrm{g} / \mathrm{L}^{-1}\right)$. This 'wetland' system dominated by aquatic vegetation and less open water did not produce algal blooms.

The Bridgewater Creek 'pond' system with larger open water areas was prone to algal blooms. Huge variations in chlorophyll-a occurred in both Dry and Wet (Table 8); mean values exceeded WQO's. Large algal blooms periodically occurred in Ponds 1 and 2 during Dry, but chlorophyll-a was reduced in Ponds 3 to 6 . Numerous microcustaceans, in particular cladocerans, found in Ponds 2 to 6 , would have been active predators on the phytoplankton.

In Wet (storm event) lower chlorophyll-a values in Pond1 probably reflect dilution, whereas the higher chlorophyll-a in Ponds 2 to 6 compared with Pond1, appear to be a flushing-out effect.

\subsection{Macroinvertebrates and mosquitoes}

Both systems had greater macroinvertebrate species richness compared to the upstream concrete channels (Table 9). The constructed stormwater wetlands at Golden Pond had even more taxa than the natural Kameruka Wetlands downstream. The Bridgwater Creek stormwater ponds recorded 86 species over a 4 year period.

Neither constructed stormwater treatment system supported successful mosquito breeding. Table 10 shows less than $5 \%$ of dips over a 12 month period conducted during Year 2 contained mosquito larvae and when present, they were in very low numbers. Larvae generally occurred amongst dead vegetation and most were only the very juvenile 1 st and 2 nd instars. No pupae were found indicating that the larvae did not complete their lifecycle. Microcustaceans, dragonfly nymphs, hemipterans (water bugs, water boatman and surface striders), water beetle larvae and adults, are known predators of mosquito larvae (Greenway et al., 2003).

\section{Discussion}

\subsection{Water quality}

The reduction of suspended solids in a wetland due to settling shows an exponential development over time - first order kinetic decay model (k-C* model) (Kadlec and Wallace, 2009). The amount of 'decay' depends upon the retention time within the wetland or pond, however this never reaches zero due to 'background' or irreducible concentrations $\left(\mathrm{C}^{*}\right)$. Similarly reductions in nitrogen and phosphorus can be explained by the $\mathrm{k}^{-\mathrm{C}^{*}}$ model. Kadlec and Wallace (2009) have identified irreducible concentrations for a range of contaminants.

Kasper and Jenkins (2007) report on an extensive daily sampling program at Bridgewater Creek wetland. Following a storm event significant reductions in TSS occurred within $24 \mathrm{~h}$ however it took $72 \mathrm{~h}$ for TSS to decay to $\mathrm{C}^{*}$. TSS in the outlet Pond 6 was only lower than in the sediment basin Pond1 during storm events, confirming results presented in this paper. They attributed increases in TSS in dry weather to 3 factors: poor design of the inlet structure between Pond 1 and Pond 2 causing a zone of recirculation and turbulence; lack of macrophytes (aquatic vegetation) in Ponds2, 3, 4 reducing filtration/settlement and sediment stabilisation (Greenway, 2004); water bird activity causing resuspension in Ponds2, 3, 4. Greenwaythis paper (Table 8) also found high chlorophyll-a in Pond2 which would contribute to TVS.

Lack of macrophytes can be related to both poor design and lack of maintenance (Greenway et al., 2007; Hunt et al., 2011). Water bird activity can be related to anthropogenic influence since local residents and visitors commonly feed the ducks and ibis from the board walk located between Ponds2, 3, 4. The viewing platform and benches overlooking Pond3 is a particularly popular spot for bird feeding as observed by Kasper and Jenkins (2007) and Greenway.

Table 9

Macroinvertebrate taxa in Golden Pond and Bridgewater Creek systems. *Indicates taxa that are predators of mosquito larvae.

\begin{tabular}{|c|c|c|c|c|c|}
\hline \multirow{2}{*}{$\frac{\text { Macroinvertebrate taxa }}{\text { Order \& number of species }}$} & \multicolumn{3}{|l|}{ Golden pond } & \multicolumn{2}{|l|}{ Bridgewater Creek } \\
\hline & $\begin{array}{l}\text { Concrete channel } \\
\text { upstream }\end{array}$ & $\begin{array}{l}\text { Stormwater } \\
\text { wetlands } 1 \& 2\end{array}$ & $\begin{array}{l}\text { Natural wetland } \\
\text { downstream }\end{array}$ & $\begin{array}{l}\text { Concrete channel } \\
\text { upstream }\end{array}$ & $\begin{array}{l}\text { Stormwater } \\
\text { ponds 1-6 }\end{array}$ \\
\hline Annelida, Nematoda, Flatworms ('worms') & 3 & 6 & 6 & 3 & 9 \\
\hline Gastropoda (water snails) & 5 & 8 & 8 & 4 & 5 \\
\hline Copepoda, Ostracoda, Cladocera*(microcustaceans) & 3 & 5 & 4 & 1 & 8 \\
\hline Acarina*(water mites,spiders) & 3 & 5 & 1 & & 6 \\
\hline Epiproctophora* (dragonflies) & 5 & 11 & 5 & 6 & 6 \\
\hline Zygoptera (damselflies) & 1 & 5 & 5 & 1 & 5 \\
\hline Ephemeroptera (mayflies) & 1 & 1 & 1 & 1 & 1 \\
\hline Hemiptera* (water bugs, water boatmen, striders) & 1 & 6 & 2 & 2 & 15 \\
\hline Diptera (flies, mosquitoes) & 2 & 3 & 2 & 6 & 10 \\
\hline Coleoptera*(water beetles) & 1 & 4 & 4 & 3 & 19 \\
\hline Trichoptera (caddis flies) & & 1 & 1 & & 2 \\
\hline TOTAL TAXA & 25 & 55 & 39 & 27 & 86 \\
\hline
\end{tabular}

Table 10

Relative abundance of mosquito larvae at Golden Pond (Wetlands 1 \&2) and Bridgewater Creek (Ponds 1-6) in Year 2 following construction.

\begin{tabular}{|c|c|c|c|c|c|}
\hline Location & Number of dips & $\%$ With larvae & $\%<10$ larvae & $\%>1-40$ larvae & \% Pupae \\
\hline Golden Pond & 130 & 4.8 & 4.0 & 0.8 & 0 \\
\hline Bridgewater Creek & 220 & 5.0 & 4.6 & 0.4 & 0 \\
\hline
\end{tabular}


Table 11

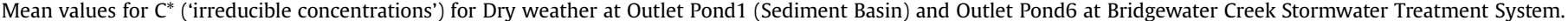
(Source; Kasper and Jenkins, 2007). Compared to this study and Event Mean Concentrations: EMC (GCWWE, 2008) and Water Quality Objectives: WQO (BCC, 2000).

\begin{tabular}{|c|c|c|c|c|c|c|c|c|c|c|c|}
\hline \multirow[b]{2}{*}{ Pond 1 out } & \multicolumn{2}{|c|}{$\mathrm{TSS} \mathrm{mg} / \mathrm{L}$} & \multicolumn{2}{|c|}{ TVS mg/L } & \multicolumn{2}{|c|}{$\mathrm{TN} \mathrm{mg} / \mathrm{L}$} & \multirow{2}{*}{$\frac{\mathrm{NH}_{4}}{\text { na* }^{*}}$} & \multirow{2}{*}{$\frac{\mathrm{NO}_{\mathrm{x}}}{\mathrm{na}^{*}}$} & \multicolumn{2}{|c|}{$\mathrm{TP} \mathrm{mg} / \mathrm{L}$} & \multirow{2}{*}{$\frac{\mathrm{PO}_{4}}{\mathrm{na}^{*}}$} \\
\hline & $8.99 *$ & 10 & $6.61^{*}$ & 7 & $1.255^{*}$ & 1.28 & & & $0.207^{*}$ & 0.22 & \\
\hline Pond 6 out & $13.69 *$ & 16 & $6.00^{*}$ & 7 & $1.041^{*}$ & 1.04 & na* & na* & $0.191^{*}$ & 0.17 & na* \\
\hline EMC: GCWWE 2008 & 19 & & na & & 1.21 & & na & 0.20 & 0.58 & & na \\
\hline WQO:BCC 2000 & 15 & & na & & 0.65 & & 0.35 & 0.13 & 0.07 & & 0.35 \\
\hline
\end{tabular}

Signs have now been erected to advise the public "Do Not Feed The Birds".

Kasper and Jenkins (2007) determined $C^{*}$ for suspended solid and nutrients in the different ponds. Table 11 presents the Pond 1 and Pond6 outlet values. Hathaway and Hunt (2010) summarised data for outflow 'irreducible concentrations' for storm water wetland basins produced from the International Stormwater BMP Database by Geosyntec Consultants and Wright water Engineers, Inc (GCWWE, 2008). The data for both Golden Pond and Bridgewater Creek are within these values for dry weather, confirming 'irreducible concentrations' ie any further reductions in TSS,TN and TP could not be expected. In storm events only TSS exceeded these 'irreducible concentrations'.

However the Water Quality Objectives (WQO) proposed by Brisbane City Council (BCC, 2000) for stormwater quality are very stringent and fail to recognise the concept of 'irreducible concentrations' for nutrients in wetland systems. Thus, it is not surprising that neither the Golden Pond nor the Bridgewater Creek stormwater wetland treatment systems met the desired WQO's for nutrients.

Hathaway and Hunt (2010) investigated the performance of 3 stormwater wetland cells (total $0.32 \mathrm{ha}$ ) in series in a small 12.5 ha watershed in North Carolina USA. For the storm events monitored final mean outlet 'irreducible' concentrations were $9 \mathrm{mg} / \mathrm{L} \mathrm{TSS}$; $0.07 \mathrm{mg} / \mathrm{L} \mathrm{NO}_{\mathrm{x}} ; 0.72 \mathrm{mg} / \mathrm{L} \mathrm{TN}$; and $0.10 \mathrm{mg} / \mathrm{L} \mathrm{TP}$ similar to Golden Pond and Bridgewater Creek with the exception of TSS. Hathaway and Hunt also investigated nitrogen speciation and noted an increase in Total Kjeldahl Nitrogen (ammonium and organic nitrogen ON) between Cell 2 and Cell3 which they attributed to organic N with an 'irreducible' concentration of $0.65 \mathrm{mg} / \mathrm{L}$. At Golden Pond and Bridgewater Creek outlets ON was 0.58 and $0.61 \mathrm{mg} / \mathrm{L}$ respectively ie similar 'irreducible' concentration. However ON was very high $2.10 \mathrm{mg} / \mathrm{L}$ at Pond 1 outlet and progressively reduced in concentration as the stormwater flowed through the wetland ponds.

Increases in $\mathrm{NH}_{4}-\mathrm{N}$ within wetlands can be attributed to the ammonification of accumulated in-situ organic matter coupled with lower sediment interface oxygen, thereby limiting nitrification (Kadlec and Wallace, 2009). Bayley (2007) also reported export loads of $\mathrm{NH}_{4}-\mathrm{N}$ between Pond1 and Pond6 at Bridgewater Creek wetland. During the winter dry months he noted that $\mathrm{NH}_{4}-\mathrm{N}$ dominated the TN pool which he attributed to mineralisation of Organic N; lower redox potential (limiting nitrification) and a lower phytoplankton biomass (limiting biological uptake). Hathaway and Hunt (2010) recorded increased organic N and TKN in Wetland Cell 3 indicating in-situ release of $\mathrm{NH}_{4}-\mathrm{N}$.
Moore et al. (2011) compared ON and TN fractions and removal in 7 stormwater wetlands in North Carolina and found ON outlet concentrations ranged from 0.7 to $0.8 \mathrm{mg} / \mathrm{L}$. They found no distinction between dry and wet weather samples. In both North Carolina and Brisbane wetlands ON outlet concentrations were significantly less than inflow stormwater runoff, thus despite irreducible concentrations, constructed stormwater wetlands are effective in reducing ON exports downstream. These 'irreducible' concentrations for organic $\mathrm{N}$ in stormwater wetland outlets, gives further credence to the rather stringent Water Quality Objective of $0.65 \mathrm{mg} / \mathrm{L} \mathrm{TN}$ in the BCC (2000) WQO guidelines.

Outflow ON: TN ratios ranged from 0.33 to 0.98 with a median value of 0.75 , again comparable to the 2 Brisbane wetlands. However the mean $\mathrm{ON}$ : TN ratio for stormwater runoff was 0.65 which differed from Golden Pond 0.26 and Bridgewater Creek 0.85 in storm events. In dry events only $2 \%$ of TN was ON (93\% being NOx) flowing into Golden Pond. The range of values reflects differences in catchment characteristics.

Moore et al. (2011) suggest that 'threshold effluent concentrations' should be considered as a more meaningful metric for nitrogen performance since \% removal does not account for 'irreducible' background concentrations.

\subsection{Macroinvertebrates}

The creation of these stormwater wetlands from concrete drainage channels has provided habitats for a wide range of macroinvertebrates. Although not quantitatively measured, other wild life associated with both systems included frogs, reptiles and birds.

Herrmann (2012) monitored macroinvertebrate colonisation over 3 years in a 1 ha constructed stormwater wetland in Kalmar, Sweden. The highest number of taxa (55 species) was found in year1 with the greatest diversity of Coleoptera (22spp) and Hemiptera (11spp), but taxa decreased to 36 with Diptera (9spp) having the greatest diversity. These changes in community composition were attributed to the early colonisation ability of beetles and corixids into the more open water body. At Bridgewater Creek, Coleoptera (19spp) and Hemiptera (15spp) as well as microcustaceans, were also the most diverse group due to the large expanse of open water in the ponds. However dense littoral vegetation also provided a habitat for many taxa, resulting in an increase in total taxa over the 4 years (Greenway and Polson, 2007). Thus the Bridgewater Creek pond system provided both open water and

Table 12

Social attributes and opportunities associated with the Golden Pond and Bridgewater Creek Constructed Stormwater Wetland Systems.

\begin{tabular}{|c|c|c|}
\hline Attribute/opportunity & Golden pond system & Bridgewater Creek system: Bowie's Flat wetland \\
\hline Recreation & Walking trails; linkages; bird watching & Walking trails; boardwalks; linkages; observation decks; bird watching \\
\hline Education Research & Interpretive signage; website & Interpretive signage; brochures; websites; used by schools; universities \\
\hline Landscape & $\begin{array}{l}\text { Open water; aquatic plants; terrestrial } \\
\text { vegetation; picnic table/shelter; car parking }\end{array}$ & $\begin{array}{l}\text { Open water; aquatic plants; terrestrial vegetation; seats/benches; picnic } \\
\text { tables/shelters; car parking water storage/irrigation }\end{array}$ \\
\hline $\begin{array}{l}\text { Community } \\
\text { Engagement }\end{array}$ & & Community catchment group; community working bees; educational workshops \\
\hline
\end{tabular}


vegetated habitats compared to the more densely vegetated wetland at Golden Pond.

Moore and Hunt (2012) quantitatively assessed macroinvertebrates in 20 stormwater wetland and 20 stormwater ponds in North Carolina, USA. They quantified biodiversity in terms of species richness (families) and found that wetlands and ponds supported similar diversity but found differences in community composition. Wetlands, and ponds with 'vegetated shelves' (littoral vegetation), supported a greater proportion of mosquito predators and a lower frequency of mosquito larvae. These findings support this study and the previous work of Greenway et al. (2003), Greenway and Polson (2007) which identified the importance of aquatic plant habitat for macroinvertebrate diversity. With habitat, rather than water quality, influencing total species richness.

\subsection{Social values}

The construction of stormwater treatment wetlands in these existing residential suburbs by retrofitting the concrete drains has enhanced social services and the landscape amenity by providing blue and green open space (Table 12). Both wetlands offer a range of recreational and educational attributes and opportunities, however the Bridgewater Creek/Bowie's Flat Wetland provides the greatest opportunities. Brisbane City Council, the Norman Creek Catchment Coordinating Committee, bird watching and bushwalking community groups all promote activities. Additionally the rainwater runoff 'stored' in the wetlands provides an opportunity for stormwater harvesting and is reused to irrigate the surrounding landscape.

Moore and Hunt (2012) qualitatively assessed 'cultural services 'of stormwater wetlands and ponds using a rubric framework with scores for similar social attributes. Based on their scoring criteria, Bridgewater Creek would achieve the highest score 4/4 for recreation and education and Golden Pond 1/4. The wide range of social befits at the Bridgewater Creek: Bowie's Flat Wetland can be attributed to community consultation in the planning stage involving Brisbane City Council, the Bridgewater Creek Community Reference Group and the Norman Creek Catchment Coordination Committee. In recognition, Brisbane City Council received a Healthy Waterways Government Award for the 'Bridgewater Creek Water Quality Improvement Project' (Water by Design-Bridgewater Creek Wetland).

\section{Conclusions}

These 2 retrofit stormwater wetlands constructed from concrete drainage channels improved water quality and created aquatic biodiversity in an urbanised setting. Both wetlands had a similar catchment size (180 ha) however water quality monitoring demonstrated that the larger 0.8 ha Bridgewater Creek system with a $12,500 \mathrm{~m}^{3}$ storage capacity was more effective in reducing suspended solids during storm events. This was also attributed to the design which incorporated an overflow bypass channel. At Golden Pond high flow-through velocities caused by the v-notch weir prevented settling and resuspended sediment. During dry weather duck activity in both wetlands caused resuspension of sediment. Both wetlands were effective in removing nutrients; however outflow concentrations did not meet Water Quality Objectives due to 'irreducible' background concentrations. This suggests that Brisbane City Council's stormwater quality objectives are too stringent for constructed wetland stormwater treatment devices.

Monitoring water quality in the natural Kameruka Wetlands demonstrated the importance of retaining and incorporating any existing wetlands as a component of a stormwater treatment chain.
Open water and dense littoral vegetation provided macroinvertebrate habitats. Macroinvertebrate assemblages in the constructed wetlands were higher than the concrete drainage channels; and even higher than in the Kameruka Wetlands.

Both constructed wetland systems provided green and blue infrastructure not only to improve stormwater quality but to create an environment for nature and people. Community and stakeholder engagement in the planning stage of the Bridgewater Creek wetland ensured the greatest opportunities; transforming a featureless open space dissected by a concrete drain into a wildlife haven and a major community attraction.

\section{Acknowledgements}

This project was funded through the Cooperative Research Centre for Catchment Hydrology, and the School of Environmental Engineering, Griffith University. The following staff and students have contributed to the monitoring program and data presented in this paper: Graham Jenkins, Carolyn Polson, Nicole Le Muth, Anu Datta and Thomas Kasper.

\section{References}

APHA, AWWA and WEF, 2005. Standard Methods for the Examination of Water and Wastewater, twenty-first ed. American Public Health Association, Washington, DC.

Bayley, M.L., 2007. Constructed Ponds for the Treatment of Urban Stormwater Biotic Processes Influencing the Removal of Nitrogen, Phosphorus and Carbon (PhD thesis). Griffith University.

Barbarosa, A.E., Fernandes, J.N., David, L.M., 2012. Key issues for sustainable urban stormwater management. Water Res. 46, 6787-6798.

Brisbane City Council, 1999a. Bridgewater Creek Stormwater Treatment System Feasibility Study, City Design, Brisbane, Australia.

Brisbane City Council, 1999b. Urban Stormwater Management Strategy for Brisbane City Council, Version2nd edition (Brisbane, Australia).

Brisbane City Council, 2000. Guideline on Identifying and Applying Water Quality Objectives in Brisbane City. Brisbane City Council, Brisbane.

Cereghino, R., Boix, D., Cauchie, H., Martens, K., Oertili, B., 2014. The ecological role of ponds in a changing world. Hydrobiologia 723, 1-6.

Geosyntec Consultants and Wright water Engineers, Inc (GCWWE), 2008. In: Hathaway, J.M., Hunt, W.F. (Eds.), Analysis of Treatment Systems Performance: International Stormwater Best Management Practices (BMP) Database (1999-2008) (2010)

Greenway, M., 2004. Constructed wetlands for water pollution control - processes, parameters and performance. Dev. Chem. Eng. Min. Process. 12 (5/6), 1-14.

Greenway, M., Dale, P., Chapman, H., 2003. An assessment of mosquito breeding and control in 4 surface flow wetlands in tropical-subtropical Australia. Water Sci. Technol. 48 (5), 249-256.

Greenway, M., Polson, C., 2007. Protecting Aquatic Ecosystem Health: Are Water Quality Objectives Realistic? Case Studies from Queensland, Australia, vol. 3. NOVATECH, pp. 1747-1754.

Greenway, M., Jenkins, G., Polson, C., 2007. Macrophyte Zonation in Stormwater wetlands-getting it right! A case study from subtropical Australia. Water Sci. Technol. 56 (3), 223-231.

Hathaway, J.M., Hunt, W.F., 2010. An evaluation of stormwater wetlands in series in Piedmont, North Carolina. J. Environ. Eng. 136, 140-146.

Herrmann, J., 2012. Chemical and biological benefits in a constructed stormwater wetland in Kalmar, S.E. Sweden. Limnologica 42, 299-309.

Hunt, W.F., Greenway, M., Moore, T.C., Brown, R.A., Kennedy, S.G., Line, D.E., Lord, W.G., 2011. Constructed storm-water wetland installation and maintenance: are we getting it right? J. Irrig. Drainage Eng. 137 (8), 469-474.

Hvitved-Jacobsen, T., Vollertsen, J., Nielsen, A.,H., 2010. Urban and Highway Stormwater Pollution: Concepts and Engineering. CRC Press, Taylor \& Francis Inc, Boca Raton, Fl, p. 347.

Kadlec, R., Wallace, S., 2009. Treatment Wetlands, CRC Press, Boca Raton, FL

Kasper, T.M., Jenkins, G.A., 2007. Measuring the background concentration in a constructed stormwater treatment wetland. Urban Water J. 4 (2), 79-91.

Marsalek, J., Schreier, H., 2009. Innovation in stormwater management in Canada: the way forward. Overview of the theme issue. Water Qual. Res. J. Can. 44 (1) v$\mathrm{x}$.

Martin, C., Ruperd, Y., Legret, M., 2007. Urban stormwater drainage management: the development of a multicriteria decision aid approach for best management practices. Eur. J. Oper. Res. 181, 338-349.

Mitsch, W.J., Gosselink, J.G., 2007. Wetlands, fourth ed. Wiley.

Moore, T.L.C. Hunt, W.F., Burchell, M.R., Hathaway, J., 2011. Organic nitrogen exports from urban stormwater wetlands in North Carolina. Ecol. Eng. 37, 589-594.

Moore, T.L.C., Hunt, W.F., 2012. Ecosystem service provisions by stormwater wetlands and ponds- a means for evaluation? Water Res. 46, 6811-6823. 
Ramsar Information Sheet: Moreton Bay http://www.environment.gov.au/water/ topics/wetlands/database/pubs/41-ris.pdf.

Roy, A.H., Wenger, S.J., Fletcher, T.D., Walsh, C.J., Ladson, A.R., Shuster, W.D., Thurston, H.W., Brown, R.R., 2008. Impediments and solutions to sustainable watershed-scale urban stormwater managements: lessons learnt from Australia and the United States. Environ. Manag. 42, 344-359.

Tixier, G., Lafont, M., Grapentine, L., Rochforte, Q., Marsalek, J., 2011. Ecological risk assessment of urban stormwater ponds: literature review and proposal of a new conceptual approach providing ecological quality goals and the associated bioassessment tools. Ecol. Indic. 11, 1497-1505.

US EPA, 1996. Overview of the Storm Water Program (EPA 883-R-96-008). Office of Water, EPA, Washington, DC.
US EPA, 2000. Low Impact Development - a Literature Review (EPA 841-B-00-005) Office of Water, EPA, Washington, DC.

Water by Design-Bridgewater Creek Wetland: http://waterbydesign.com.au/ bridgewater-creek-wetland/.

Whelans, C., Maunsell, H.G., Thompson, P., 1994. Planning and Management Guidelines for Water Sensitive Urban (Residential) Design. Dept. Planning and Urban Development, Western Australia, Perth, WA.

Wetzel, R.G., 2001. Limnology: lake and river ecosystems, third ed. Academic Press. Woodcock, T.S., Monaghan, M.C., Alexander, K.E., 2010. Ecosystem characteristics and summer secondary production in stormwater ponds and reference wetlands. Wetlands 30, 461-474. 